\section{Research integrity guidelines in Japan}

It is to be hoped that Japan's new guidelines for research integrity, released recently by the Ministry of Education, Culture, Sports, Science and Technology (MEXT), will curb research misconduct (see T. Tanimoto et al. Nature 512, 371; 2014).

Institutions in Japan have previously tended to avoid taking responsibility for misconduct by their scientists. Under the revised guidelines, a research institution must take appropriate measures against any scientist who is found guilty of data manipulation or fabrication, for example. Should it fail to do so, MEXT will cut its research budget.

MEXT has already reduced RIKEN's requested budget for next year by nearly $20 \%$ (¥12.1 billion; US\$111 million) as a penalty for inefficient handling of the two 'STAP' stem-cell papers published and subsequently retracted this year (see Nature 511, 112; 2014). Masanori Wada Tokyo Institute of Technology, Japan. wada.m.ae@m.titech.ac.jp

\section{Lung-cancer screens now worth the cost}

False-positive results from computed-tomography (CT) scans were a cause for concern in the 2011 US National Lung Screening Trial (see Nature 513, S4-S6; 2014). But false-positives have now been cut significantly owing to improved imaging technology and more-refined screening protocols (see B. J. McKee et al. J. Am. Coll. Radiol. http://dx.doi.org/10.1016/j. jacr.2014.08.002; 2014).

Contrary to your implication, an actuarial analysis indicates that CT lung scanning is cost-effective (see B. Pyenson et al. Am. Health Drug Benefits 7, 272-282; 2014) in the US population covered by the health-insurance programme
Medicare. Most members screened are aged over 65 .

The study finds that the average monthly cost of CT lung scanning per Medicare member is just US\$1 (the equivalent screening cost for breast and colorectal cancers is $\$ 2.50$ and $\$ 1.40$, respectively).

This latest cost-benefit analysis is consistent with other peerreviewed research proving that lung-cancer screening is costeffective for Medicare and for private payers too.

As James Mulshine, a translational-medicine specialist at Rush University in Chicago, Illinois, pointed out in a 2010 Lung Cancer Alliance statement, through screening, "we have the opportunity to realize the greatest single reduction of cancer mortality in the history of the war on cancer" (see go.nature.com/vs2smt).

Laurie Fenton-Ambrose Lung Cancer Alliance, Washington DC, USA.

rryan@powelltate.com

Ella A. Kazerooni American

College of Radiology,

Washington DC, USA.

\section{Protect privacy of mobile data}

The use of new data sources to model humans' behavioural responses to climate change (see P. Palmer and M. Smith Nature 512, 365-366; 2014) raises methodological and ethical issues.

The authors do not mention the importance of call-detail records (CDRs), normally collected by mobile-phone operators for commercial purposes. Compared with data collected by smartphones from global-positioning systems, CDRs have lower location accuracy and differ in their potential for modelling and privacy risks.

The poorest communities in low- and middle-income settings should be at the centre of modelling efforts because they are among the most vulnerable to climate change.

These populations are unlikely to use satellite navigation and social media, and they might not conform to human-mobility models derived from commuting patterns in wealthy countries. In these regions, CDRs can provide important insight - if appropriate privacy protections are in place (see, for example, A. Wesolowski et al. Science 338, 267-270; 2012).

The rights of the individual to control their private data and the needs of researchers and policy-makers to access data for societal good create tensions that are central to the effective modelling of human behaviour (see, for example, Y. de Montjoye et al. Sci. Rep. 3, $1376 ; 2013)$. These call for new regulatory and institutional review board processes.

Caroline O. Buckee ${ }^{\star}$ Harvard School of Public Health, Boston, Massachusetts, USA; and Flowminder Foundation. cbuckee@hsph.harvard.edu ${ }^{*}$ On behalf of 5 correspondents (see go.nature.com/pezxek for full list.)

\section{Invest in renewable energy in Tibet}

Tibet's fragile environment is being damaged by a paucity of energy, as well as by pollutants and litter (see Nature 512, 240241; 2014). Greater investment could unleash the region's huge potential to produce renewable energy.

Access to fossil fuels is extremely limited in Tibet, particularly in rural areas. Biomass - including manure, firewood and crop residues is largely used instead, making up two-thirds of total energy use. However, this degrades forest and grassland and causes indoor pollution (see G. Liu et al. Renew Sust. Energ. Rev. 12, 1890-1908; 2008).

Tibet has abundant resources for renewable energy (including solar, wind and geothermal) owing to its complex topography and widely varying climate (see L. Shen et al. Environ. Manage. 46, 539-554; 2010).

These resources remain mostly untapped, however, because of the high cost of exploitation, unevenly distributed settlements, lack of local infrastructure, and inadequate maintenance and knowledge.

Gang Liu Norwegian University of Science and Technology, Trondheim, Norway. geoliugang@gmail.com Mario Lucas Bochum University of Applied Sciences, Bochum, Germany.

\section{Focus on positive features of ageing}

Ageing is not just a linear physiological decline (see L. Fontana et al. Nature 511, 405-407; 2014). Research into its more positive features could lead to a better-quality and longer life.

Personal resources such as optimism, resilience and engagement are integral to ageing well (see, for example, T. D. Cosco et al. BMJ Open 3, e002710; 2013).

Investigating the contribution of psychosocial strengths to positive ageing in human models would provide further insight into, and complement, physiological animal models.

Theodore D. Cosco, Carol Brayne University of Cambridge, UK. tdc33@medschl.cam.ac.uk Blossom C. M. Stephan Newcastle University, UK.

CONTRIBUTIONS Correspondence may be submitted to correspondence@nature. com after consulting the author guidelines at go.nature.com/cmchno. 